\title{
StreetlightSim: A Simulation Environment to Evaluate Networked and Adaptive Street Lighting
}

\author{
Sei Ping Lau, Geoff V. Merrett, Alex S. Weddell, Neil M. White \\ Electronics and Computer Science \\ University of Southampton, UK \\ \{sp11g11, gvm, asw,nmw\}@ecs.soton.ac.uk
}

\begin{abstract}
Sustaining the operation of street lights incurs substantial financial and environmental cost. Consequently, adaptive lighting systems have been proposed incorporating adhoc networking, sensing, and data processing, in order to better manage the street lights and their energy demands. Evaluating the efficiency and effectiveness of these complex systems requires the modelling of vehicles, road networks, algorithms, and communication systems, yet tools are not available to permit this. This paper proposes StreetlightSim, a novel simulation environment combining OMNeT++ and SUMO tools to model both traffic patterns and adaptive networked street lights. StreetlightSim's models are illustrated through the simulation of a simple example, and a more complex scenario is used to show the potential of the tool and the obtainable results. StreetlightSim has been made open-source, and hence is available to the community.
\end{abstract}

Keywords—simulation, adaptive street lighting, smart cities

\section{INTRODUCTION}

Street lighting is a ubiquitous utility that can be found in most urban areas. Effective street lighting has been shown to reduce crime and traffic collisions, and encourage socioeconomic activities during the night [1]. Despite the benefits of street lighting, sustaining its operation is a significant financial and environmental burden on local governments. According to 2006 statistics [2], electric lighting is responsible for $2650 \mathrm{TWh}$ of annual electricity consumption, leading to the emission of 1.5 billion tonnes of $\mathrm{CO}_{2}$; powering the world's 90 million street lights accounts for over $4 \%$ of this total.

In order to accommodate rapid population growth, and with urban areas housing more than half of the global population, the concept of the Smart City has been proposed. The ultimate goal of this concept is to manage the urban infrastructure and public services in a more efficient and sustainable way through information and communication technology (ICT). One of the core areas of the Smart City is the smart environment, which requires sustainable management of the environment and its limited natural resources [3]. Given that street light numbers are expected to grow by over $300 \%$ in the coming decade [4], sustaining the operation of street lighting will potentially pose even greater energy demands (and lead to increased $\mathrm{CO}_{2}$ emissions), hence energy efficient street lighting is essential.

Recent advances in light-emitting diode (LED) lighting have dramatically improved its luminous efficacy, making it a promising solution for street lighting applications. Generally,
LED lamps offer better colour rendering, and instant light once switched on, compared to the 15-minute delay before sodiumbased lamps are fully lit. LED lamps therefore permit energy savings through fine-grained temporal control of illumination, and are more cost effective due to their extended lifespan [5].

Conventionally, street lights remain fully lit across their operational hours. This can result in significant energy wastage, especially when roads are only intermittently used and hence illumination is not necessary. In the United Kingdom (UK), some local governments selectively switch off street lights in specific areas at certain hours [6]. For example, in Warwickshire, UK, some street lights are completely switched off between midnight and the early morning. This is predicted to save $£ 0.5$ million annually and reduce greenhouse gas emissions by 3,000 tonnes. Lately, the use of ICT in adaptive street lighting has attracted much attention. Remote- and sensor-controlled street lighting schemes offer further opportunities for energy saving, as they enable the continual adjustment of lighting levels. Generally, a remote and centralised control centre manages street light dimming and health monitoring [7]. In some cases, the street light operations are adaptively adjusted based on locally sensed ambient parameters, such as weather and traffic conditions [8]. Artificial intelligence based control has also been considered to optimise street light management and operation [9].

Although existing approaches have aimed to improve the sustainability of street lighting, most have focused on improving energy efficiency without considering the impact on the utility of street lighting from the road users' perspective. Simulation tools such as OMNeT++, ns-2 and JiST have been used to evaluate adaptive street lighting approaches in terms of their energy saving [1, 10] and networking performance [11]. However, we believe that effective evaluation requires simulation tools that can also evaluate the utility of street lighting schemes to road users. In this paper, we report on the StreetlightSim, a novel simulation environment that models both traffic patterns and adaptive networked street lights. StreetlightSim is open-source, and has been made available to the community at www.streetlightsim.ecs.soton.ac.uk.

\section{OVERVIEW OF STREeTlightSim}

As illustrated in Fig. 1, the main components of StreetlightSim are built within $\mathrm{OMNeT}++$ modules and Simulation of Urban MObility (SUMO), a road traffic simulator 


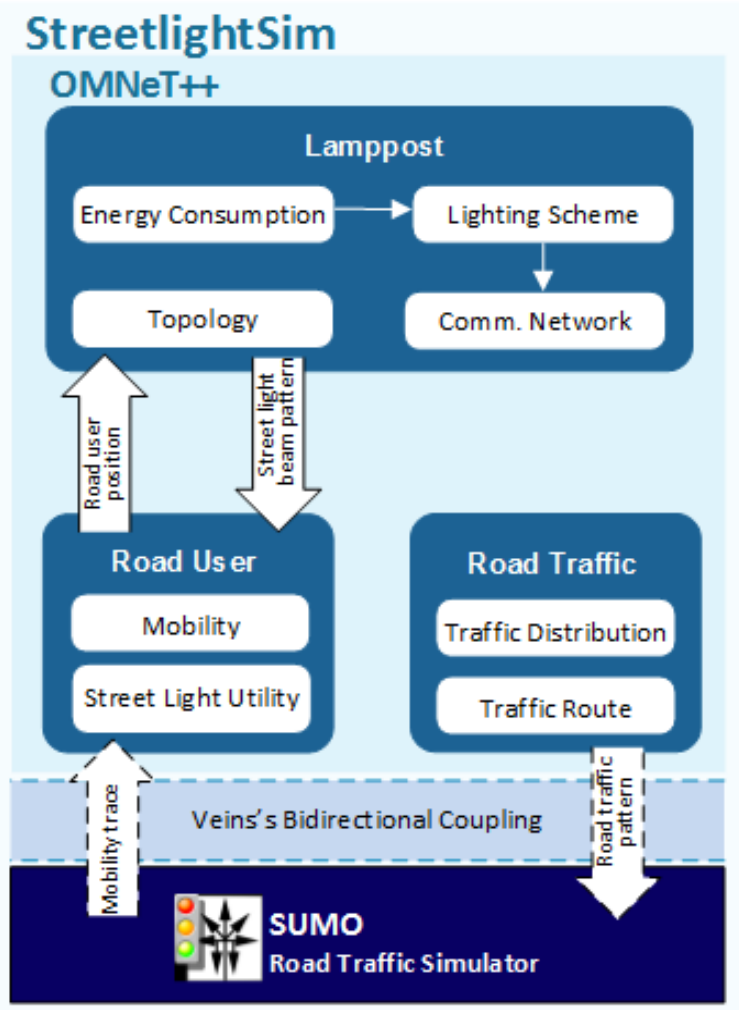

Figure 1. Block diagram of StreetlightSim

that acts as a real-time mobility trace generator, obeying specified road traffic patterns. StreetlightSim is adapted from the Vehicle in Network Simulation (Veins) framework [12], which provides a solid foundation for bidirectional coupling between OMNeT++ and SUMO. Veins also facilitates the modelling and simulation of individual vehicles, which permits the evaluation of street lighting effectiveness from the perspective of each individual road user. In addition, OMNeT++ allows the simulation of data processing and control algorithms, and communication networks - supporting frameworks and models including MiXiM, INET and INETMANET [13].

The following subsections explain the operation of StreetlightSim's components in Fig. 1.

\section{A. Bidirectional Coupling}

Traffic Control Interface (TraCI) is a client/server architecture-based interface of SUMO [14]. This interface provides an opportunity for real-time retrieval and manipulation of simulated objects, such as road users, traffic lights and street lights via a Transmission Control Protocol (TCP) connection. Veins exploits this interface using a dedicated OMNeT++ module to establish a proxy TCP connection with TraCI.

During simulation, TraCI forwards a series of commands via the established TCP connection, at regular time intervals. Upon arrival at SUMO, these commands are processed and associated values are returned to the module at the next simulation timestep. Although TraCI specifies many standard commands, the most frequently used is to retrieve the real-time mobility trace generated by SUMO. The OMNeT++ module uses the generated mobility trace as a trigger to update the simulation scenario.

\section{B. Road Traffic}

In the original Veins implementation, road traffic patterns are not explicitly specified, instead using the standard SUMO configurations such as flow or route to define a specific road traffic distribution. In Veins, it is justifiable to use such an approach as it was intended to investigate the influences of intervehicle communication in traffic flow. However, in evaluating the effectiveness of street lighting schemes, StreetlightSim requires a realistic road traffic distribution during hours of street light operation.

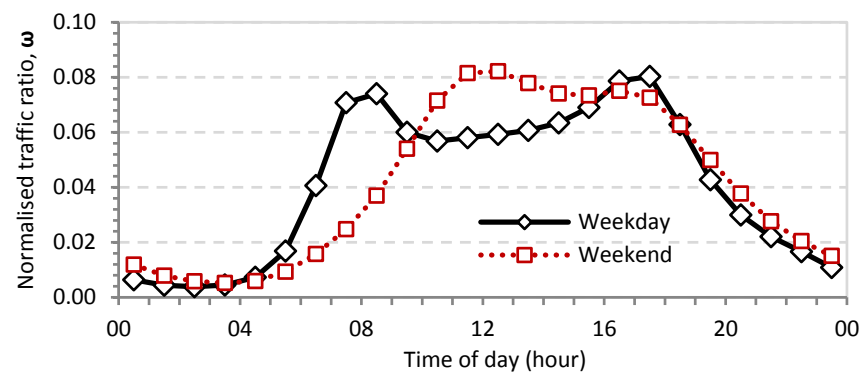

Figure 2. Traffic distribution ratio by time for the average weekend and weekday vehicular traffic (adapted from [15]).

To introduce a reasonable approximation of road traffic pattern, StreetlightSim adopts the time-dependent traffic distribution ratio shown in Fig. 2. Two traffic distribution ratios are considered, representing average weekday and weekend traffic ratios, as they exhibit significantly distinct traffic trends. These distribution ratios are multiplied by the Annual Average Daily traffic Flow (AADF) and are used to randomly inject simulated motorists as the simulation runs. The AADF represents the daily average number of vehicles passing a point in the road network, based on empirical data collected over a complete year. Although AADF only accounts for vehicular traffic, pedestrians also utilise street lights - therefore, pedestrian traffic is also introduced into the simulation. AADF with additional pedestrian traffic is shown in Eq. 1 and is assumed to share the same distribution exhibited by the vehicular traffic.

$$
A A D F_{\text {ped }}=\frac{A A D F}{1-\Delta_{\text {ped }}} ; 0 \leq \Delta_{\text {ped }}<1
$$

where $\Delta_{\text {ped }}(\%)$ is the additional pedestrian traffic composition according to the city of interest.

Since weekday and weekend have different traffic volumes, the total number of road user introduced during the simulation according to either of these days is given by:

$$
\Gamma(\theta)=\beta(\theta) A A D F_{\text {ped }}
$$

where $\theta$ represents either weekday or weekend traffic, and $\beta$ is the traffic weight of these days. In StreetlightSim, $\beta($ weekday $)=1.13$ and $\beta($ weekend $)=0.87$ is adopted. Thus, the total number of road users introduced during the simulated hour $h$ for either weekday or weekend traffic is given by:

$$
\Gamma(h, \theta)=\omega(h, \theta) \Gamma(\theta)
$$

where $\omega(h, \theta)$ is the normalised traffic ratio at hour $h$ on $\theta$, see Fig. 2. 
To inject a simulated pedestrian or motorist into SUMO through TraCI, each of these road users need to be associated with a correct path that specifies the route it travels as simulation runs. SUMO provides tools to generate these random paths, e.g. randomTrip.py and duaItera.py. However, these paths are numerically identified and are not explicitly for either pedestrians or motorists. Therefore, they are not 'StreetlightSimready'. In order to convert these random paths into a usable format, StreetlightSim includes a tool to categorise these random paths either for pedestrians or motorists.

\section{Lamppost}

StreetlightSim is based on the MiXiM framework, making it possible to analyse the effectiveness of street lighting schemes together with different wireless communication networks. As the performance of these communication networks is spatiallysensitive, their evaluation requires knowledge of real street light topologies. In StreetlightSim, the topology is derived using OpenStreetMap (www.openstreetmap.org) and JOSM (josm.openstreetmap.de). OpenStreetMap is a communitydriven free editable map of the world, and JOSM is a map editor for OpenStreetMap. The data from OpenStreetMap is used by StreetlightSim as the source map for the road network and random path generation. While it provides comprehensive data on the road network, it does not include the locations of street lights. In order to use the OpenStreetMap in StreetlightSim, the minimum enhancement to the data is to identify the relative locations of street lights within the topology. Depending on the simulation scenario, additional pedestrian footpaths could be added to the data to simulate pedestrian traffic distinct from vehicular traffic. These enhancements can be accomplished using JOSM.

To evaluate the energy efficiency of street lighting schemes, the energy model shown in Eq. 4 is assumed. This model assumes that the street light energy consumption is directly proportional to its illuminance output e.g. when the street light illuminance output is set to $80 \%$, the street light energy consumption is also reduced to $80 \%$ based on its maximum power rating $P_{\max }$.

$$
e(N)=\sum_{n=0}^{N} P_{\max } \varphi T
$$

where $e(N)$ is the energy consumed by a street light after $N$ discrete timesteps, $\varphi$ is the illuminance output of the street light $(\%), P_{\max }$ is the maximum power rating of the light source $(\mathrm{W})$, and $T$ is the duration of a single timestep $n$.

\section{Road User}

In StreetlightSim, this module has two functions: (a) represents the simulated road users in OMNeT++ which is characterised by the mobility traces generated by SUMO and (b) evaluates the effectiveness of the street lighting schemes according to latest positions of the simulated road users. Depending on the granularity of the simulation timestep, realtime mobility traces of the simulated road users are continuously returned by SUMO during simulation runs. OMNeT++ receives these mobility traces and uses them to update the simulation scenario by adding new road users, updating the existing road users to their latest position, or removing them from the simulation scenario once they have reached the end of their route.

Although several recommendations and standards exist for designing street lighting schemes for different classes of roads [16], adaptive street lighting (where street lights can dim or switch off) has been given little consideration. Thus, a street light utility model - a performance metric used to measure the effectiveness of street lighting to road users - is introduced in StreetlightSim. This utility model is synthesized based on the lighting needs of both pedestrians and motorists. The needs for street lighting from a motorist's perspective are based on two assumptions: (a) a motorist requires that only a segment of the road in front of them is illuminated and (b) that the length of this lit segment is $100 \mathrm{~m}$ (in practice this can be between $60-160 \mathrm{~m}$ dependent on the stopping distance required) [17]. Thus, the street light utility from a motorist's perspective at time $t$ is given by:

$$
U_{m o t}(t)=\frac{1}{100} \int_{0}^{100} \gamma(x, t) d x
$$

where $\gamma(x, t)$ is ratio of illuminance level at location $x$ meter ahead of a motorist at time $t$ to the required illuminance level for the specific class of road where motorist is travelling on.

For pedestrians, street lighting provides the primary light source to improve their perceived safety, and assists them in obstacle detection and avoidance after dark. To model the needs for street lighting from a pedestrian's perspective, we consider the segment of road $150 \mathrm{~m}$ before and after them. These road segments are subdivided into five illumination zones $(30 \mathrm{~m}$ segment of road per zone) where each zone requires different illuminance levels. The required illuminance levels of the nearest zones are set at $100 \%$ of illuminance level for specific class of road where pedestrian is traveling on, and gradually decreased by $20 \%$ for each zone further away from the pedestrian. Road segments that are outside these illumination zones are not required to be lit [18]. Thus, the street light utility from a pedestrian's perspective is given by:

$$
\begin{gathered}
\begin{array}{r}
U_{\text {ped }}(t)=\alpha U_{\text {ped (avoid) }}(t) \\
+(1-\alpha) U_{\text {ped }(\text { prospect })}(t)
\end{array} \\
U_{\text {ped(avoid) }}(t)=\frac{1}{10} \int_{0}^{10} \gamma(x, t) d x \\
U_{\text {ped (prospect) }}(t)=\frac{1}{300} \int_{-150}^{150} z(x, t) d x \\
z(x, t)= \begin{cases}\varepsilon(x, t), & \varepsilon(x, t) \leq 1 \\
1, & \varepsilon(x, t)>1\end{cases} \\
\varepsilon(x, t)=\frac{\gamma(x, t)}{1-0.2\left\lfloor\frac{|x|}{30}\right\rfloor},-150 \leq x \leq 150
\end{gathered}
$$

where $U_{\text {ped(avoid) }}(t)$ is the street light utility for obstacle and collision avoidance as well as recognition of faces at location $x$ meter ahead of a pedestrian at time $t, U_{\text {ped(prospect) }}(t)$ is the 
street light utility for good overview at location $x$ meter from a pedestrian at time $t, z(x, t)$ is the ratio of illuminance level at location $x$ meter from a pedestrian at time $t$ to illuminance level required at illumination zone where location $x$ is located, and $\alpha$ is the weight of time spent for obstacle and collision avoidance as well as recognition of faces. In this model, $\alpha=0.45$ is adopted [19].

Based on the proposed utility models, to achieve perfect street light utility at any moment, it requires a lit road segment of between $100 \mathrm{~m}$ and $150 \mathrm{~m}$ with the correct illuminance levels. In real environments, however, these lit road segments are occasionally unachievable due to different road geometries and street light topologies. Owing to this, StreetlightSim considers the furthest lit road segment within the required distance as the reference point for street light utility evaluation. For example, if there are only two street lights ahead of a motorist which provide $60 \mathrm{~m}$ of lit road segment, then the result is $100 \%$ street light utility.

\section{MOdEL VALIDATION}

In order to verify that the described operations of StreetlightSim are accurately implemented, a series of validations are performed. However, only noteworthy results are included due to space restrictions.

The following subsections describe the validation results of the models described in section II.

\section{A. Road Traffic Model}

To validate the road traffic model in StreetlightSim, five different AADF values are used: 180, 438, 1347, 3508 and 6554 vehicles per day. These AADF values represent the minimum, $1^{\text {st }}$ Quartile, median, $3^{\text {rd }}$ Quartile and maximum AADF values respectively of residential roads in Southampton, UK. To introduce pedestrian traffic, $\Delta_{\text {ped }}=0.14$ is also included during the validation. Fig. 3 shows the traffic distribution generated by StreetlightSim when the model is validated with 3508 vehicles per day and $\Delta_{p e d}=0.14$. This profile matches the trends defined in Fig. 2.

\section{B. Energy Model}

The energy model is validated using 112 street lights operating with Conventional, Part-night, Chronosense and Dynadimmer lighting schemes (see Table 1) [1] since their energy consumption can be numerically computed using Eq. 4. These street lights operate from 16:00 until 08:00 the next day, which represents one of the longest street light operational hours during winter months in the UK. All street lights are assumed to be equipped with $25 \mathrm{~W}$ LED light modules. According to the results generated by StreetlightSim, the total energy consumption of Conventional, Part-night, Chronosense and Dynadimmer lighting schemes are $44.8 \mathrm{kWh}, 29.4 \mathrm{kWh}, 31.4$ $\mathrm{kWh}$ and $28.8 \mathrm{kWh}$ respectively. These results are verified with those numerically computed using Eq. 4. Fig. 4 shows the results of energy consumed by all the street lights while operating on Conventional and Dynadimmer lighting schemes. The energy consumption of Conventional lighting scheme remains constant across different simulated hours, while Dynadimmer's fluctuates according to different illuminance outputs as specified

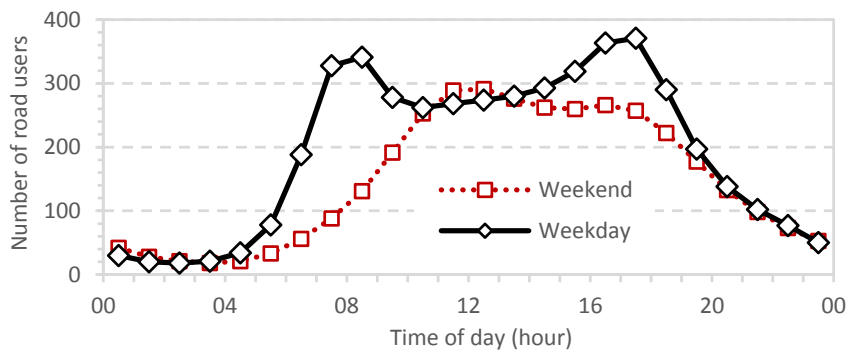

Figure 3. Simulation results showing the traffic distribution generated by StreetlightSim using AADF value of 3508 vehicles per day.

TABLE I. STREET LIGHTING SCHEMES VALIDATED IN THIS PAPER (more information can be found in[1])

\begin{tabular}{|l|l|}
\hline Lighting Scheme & \multicolumn{1}{|c|}{ Operation } \\
\hline Conventional & $\begin{array}{l}\text { All the street lights are switched on with 100\% } \\
\text { illuminance output during the simulation. }\end{array}$ \\
\hline Philips Dynadimmer & $\begin{array}{l}\text { Street lights operate at } 40 \% \text { between 23:00 and } \\
05: 00,55 \% \text { from 05:00 to 06:00 and 65\% from } \\
20: 00 \text { to 23:00 of Conventional lighting scheme } \\
\text { illuminance output. For the rest of the operational } \\
\text { hours, all the street lights operate at 90\% of } \\
\text { Conventional lighting scheme illuminance output. }\end{array}$ \\
\hline Philips Chronosense & $\begin{array}{l}\text { Similar to Conventional lighting scheme except } \\
\text { from 22:00 to 05:00 where illuminance output of } \\
\text { all the street lights is reduced to } 65 \% .\end{array}$ \\
\hline Part-night & $\begin{array}{l}\text { All the street lights are switched on with 100\% } \\
\text { illuminance output except from 00:00 to 05:30 } \\
\text { where the street lights are switched off to conserve } \\
\text { energy. }\end{array}$ \\
\hline
\end{tabular}

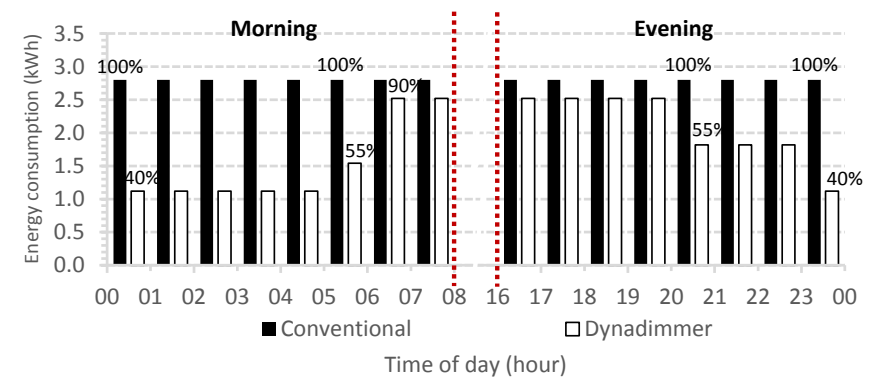

Figure 4. Simulation results showing energy consumption of Conventional and Dynadimmer lighting schemes at different street light operational hours as well as their differences in percentage.

in Table I. These results also confirm that the lighting schemes listed in Table I are implemented correctly.

\section{Utility Model}

Fig. 5 shows the lighting scenario used to validate the proposed utility models. There are eight street lights operating at $100 \%$ illuminance output while the rest are switched off. A pedestrian and a motorist are initially positioned at $x=0$, and street light utility is evaluated as they travel from left to right. The street light utility values experienced by the road users is shown in Fig. 6. Both the pedestrian and the motorist experience $100 \%$ street light utility as they travel. However, street light utility is gradually reduced to zero as they approach the unlit segment of road on the right. This trend is consistent with the lighting scenario illustrated in Fig. 5. 


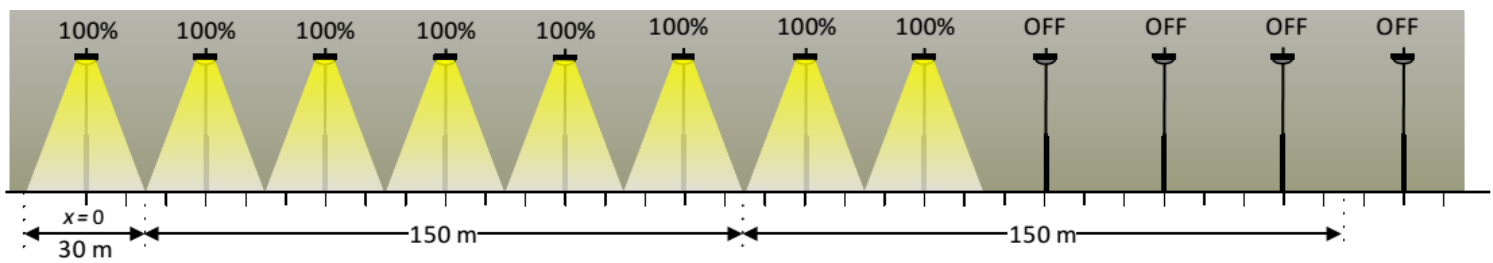

Figure 5. Lighting scenario used to validate the utility models.

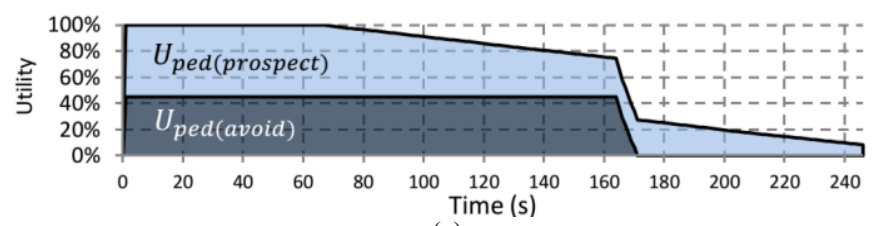

(a)

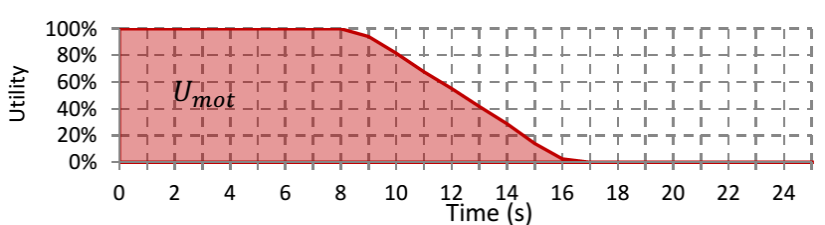

(b)

Figure 6. Simulation results showing the street light utility from (a) a pedestrian's perspective and (b) motorist's perspective while travelling right from $x=0$ under the lighting scenario shown in Fig. 5

\section{USING STREetLightSim: A CASE STUDY}

In this section, we demonstrate the potential use of StreetlightSim to analyse some factors that could affect the efficiency and effectiveness of a street lighting scheme. A street light network from a residential area located in Southampton, UK is adopted in this demonstration. This network consists of 112 street lights which scattered within approximately $3.5 \mathrm{~km}$ of residential roads. Fig. 7 summaries the process flow for using StreetlightSim; step-by-step guides are included with the downloadable version of StreetlightSim.

\section{A. Differences in Street Light Operation Period}

Street light operation periods are consistent throughout the year for those places near to the Equator. However, they are increasing seasonal further north and further south from the Equator. Although these differences are trivial for grid-based street lights, it is an important consideration for solar-harvesting autonomous street lights which have become a popular lighting solution for rural or isolated places [20]. StreetlightSim is flexible when simulating different operational hours across different seasons. For example, Fig. 8 shows the energy consumption trends of Conventional and Dynadimmer lighting schemes according to different months of year and seasonal differences in Southampton, UK.

\section{B. Road Traffic}

For time-based lighting schemes, such as Conventional, Dynadimmer, Chronosense or Part-night, street lights operate according to a predefined operation schedule, often triggered by the light sensor relay. For adaptive lighting schemes, however, the street light operations are continuously optimised according to different ambient parameters, such as traffic conditions. StreetlightSim is equipped with a road traffic model which enables any road traffic bounded lighting scheme to be analysed. For example, Fig. 9 shows energy consumption of Zoning lighting scheme [1] grows with increasing AADF values. Although this traffic model is based on UK traffic distributions, it can be easily amended to any traffic distribution for the area of interest.

\section{Targeted User}

There are some lighting schemes where a specific group of

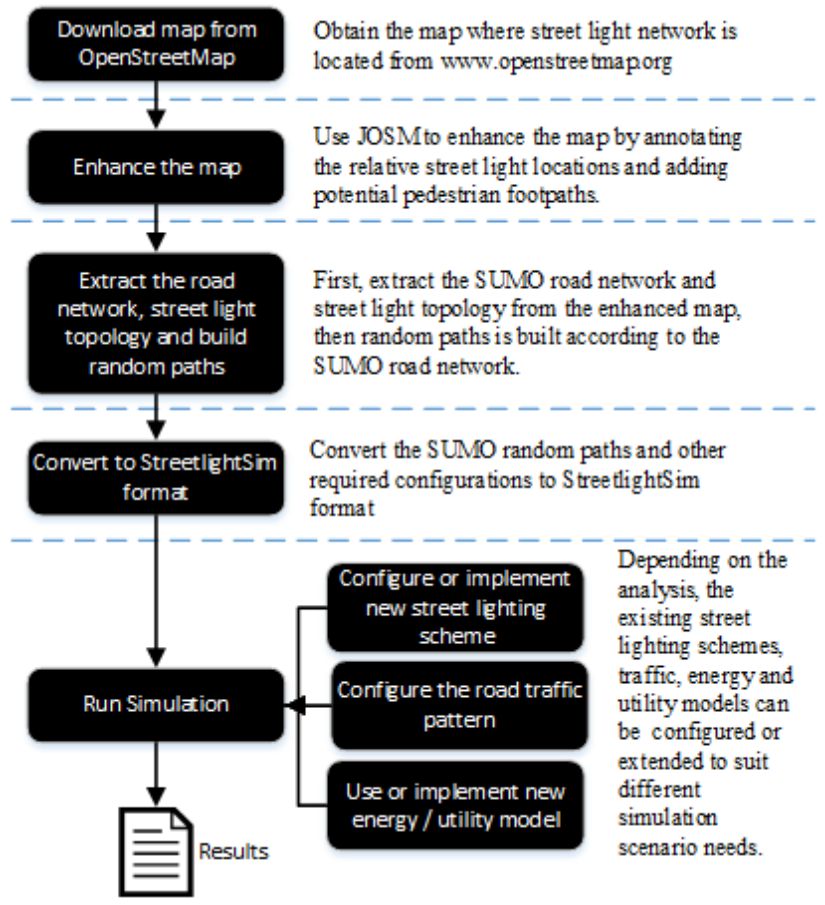

Figure 7. Process flow for using StreetlightSim.

users is targeted. With StreetlightSim, the effectiveness of lighting schemes can be analysed from different road users' perspectives using the proposed utility models. For example, the effectiveness of the Zoning lighting scheme [1] in a street light network located within a residential area is shown in Fig. 10. Since this lighting scheme is specifically designed for pedestrians, motorists derive little benefit from this lighting scheme.

\section{Communication Network}

StreetlightSim utilises the MiXiM framework. Since this framework supports many mobile and fixed wireless network models, StreetlightSim is capable of analysing street lighting schemes that operate over these networks. However, it is essential to consider the requirements of this framework while extending the simulation scenario to these networks. Fig. 11 shows the deviation of annual energy consumption of an 
adaptive lighting scheme when it is implemented using an IEEE 802.15.4 communication network compared to an ideal scenario. In the ideal scenario, this lighting scheme is capable of creating lighting conditions that provide perfect street light utility by tracking the road users via global positioning system, and an error- and delay-free communication network is adopted.

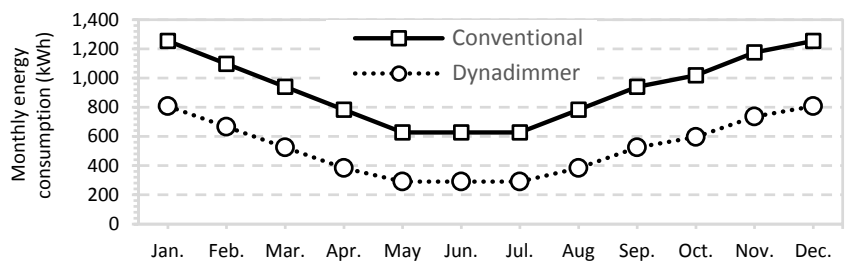

Figure 8. Simulation results showing monthly energy consumption of 112 street lights according to different months of year while operating on Conventional and Dynadimmer lighting schemes

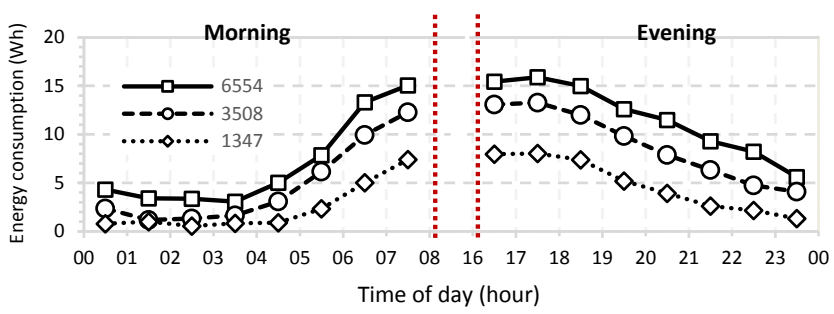

Figure 9. Simulation results showing average energy consumption of Zoning lighting scheme according to different AADF values and weekday traffic distribution.

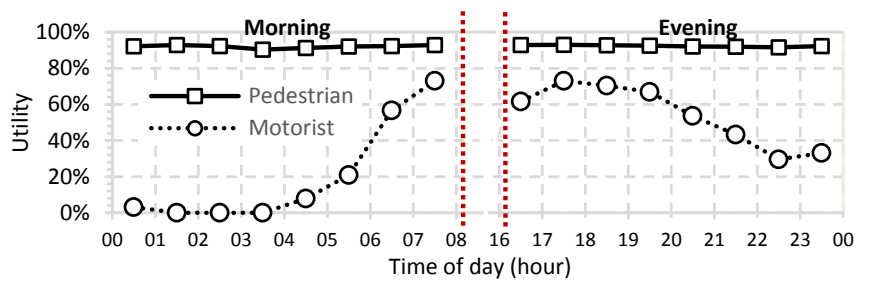

Figure 10. Simulation results showing street light utility (median) experienced by different road users while street lights operating on Zoning lighting scheme.

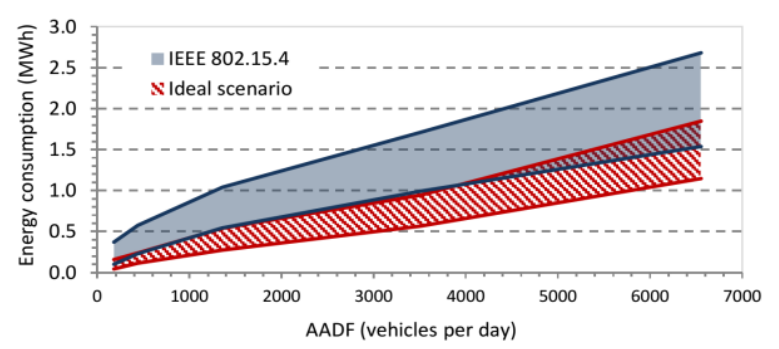

Figure 11. Simulation results showing annual energy consumption of 112 street lights according to different AADF values while operating an adaptive lighting scheme in an ideal scenario compared to IEEE 802.15.4 implementation.

\section{CONCLUSIONS}

This paper has presented StreetlightSim, a novel simulation environment modelling both traffic patterns and adaptive networked street lights. The operation of the tool has been illustrated through a realistic case study demonstrating the results that can be obtained. Our current and future research is using this simulation environment to develop new adaptive street lighting schemes. StreetlightSim is freely available to download from www.streetlightsim.ecs.soton.ac.uk.

\section{REFERENCES}

[1] S. P. Lau et al., "Energy-efficient Street Lighting through Embedded Adaptive Intelligence," in Proc. Int. Conf. Advanced Logistics and Transport, Sousse, 2013, pp. 53-58.

[2] International Energy Agency, Light's Labour's Lost: Policies for Energyefficient Lighting, France: IEA, 2006.

[3] S. Pellicer et al., "A Global Perspective of Smart Cities: A Survey," in Proc. $7^{\text {th }}$ Int. Conf. Innovative Mobile and Internet Services in Ubiquitous Computing, Taichung, 2013, pp. $439-444$.

[4] Northeast Group, LLC, Global LED and Smart Street Lighting: Market Forecast (2014 - 2025), Washington: Northeast Group, LLC, 2014.

[5] Grah Lighting, "Street Lighting Technology Comparison". [Online]. Available: http://www.grahlighting.eu/en/street-lighting-technologycomparison [Accessed: Dis. 20, 2013]

[6] Warwickshire County Council, "Part-night Lighting". [Online]. Available: http://www.warwickshire.gov.uk/partnightlighting [Accessed: Nov. 11, 2013].

[7] C. Jing et al., "Design of Streetlight Monitoring and Control System Based on Wireless Sensor Networks," in Proc. $2^{\text {nd }}$ IEEE Conf. Industrial Electronics and Applications, Harbin, 2007, pp. 57-62.

[8] S. I. Hong et al., "A Development of LED-IT-Sensor Integration Streetlight Management System on Ad-hoc," in Proc. IEEE Region 10 Conf., Bali, 2011, pp. $1331-1335$.

[9] M. Moghadam and N. Mozayani, "A Street Lighting Control System Based on Holonic Structures and Traffic System," in Proc. $3^{\text {rd }}$ Int. Conf. Computer Research and Development, Shanghai, 2011, pp. 92-96.

[10] T. Yang et al., "PKU-STRAW-L: A Simulative Platform Evaluate the Power-saving Rate of the Intelligent Street Lamp System," in Proc. $9^{\text {th }}$ Int. Conf. Ubiquitous Intelligence \& Computing, Fukuoka, 2012, pp. 525532.

[11] R. Pantoni and D. Brandão, "A Confirmation-based Geocast Routing Algorithm for Street Lighting Systems," Computers \& Electrical Engineering, vol. 37, no. 6, pp. 1147-1159, Nov. 2011.

[12] C. Sommer et al., "Simulating the Influence of IVC on Road Traffic using Bidirectionally Coupled Simulators," in Proc. IEEE INFOCOM Workshops, Phoenix, AZ, 2008, pp. 1-6.

[13] OMNeT++ Network Simulation Framework, "Simulation Models". [Online]. Available: http://www.omnetpp.org/models [Accessed: Mar. $11,2014]$

[14] A. Wegener et al, "TraCI: An Interface for Coupling Road Traffic and Network Simulators," in Proc. $11^{\text {th }}$ Communications and Networking Simulation Symp., New York, NY, USA, 2008, pp.155 - 163.

[15] United Kingdom. Department for Transport, Traffic Distribution on All Roads by Time of Day in Great Britain, 2011. London: Department for Transport. [Online]. Available: https:/www.gov.uk/government/ uploads/system/uploads/attachment_data/file/208840/tra0307.xls [Accessed: Oct. 20, 2012]

[16] P. Boyce et al., "Road Lighting and Energy Saving," Lighting Research and Technology, vol. 41, pp. 245 - 260, 2009.

[17] D. A. Schreuder, Road Lighting for Safety, London: Thomas Telford Ltd., 1998.

[18] A. Haans and Y. A. de Kort, "Light Distribution in Dynamic Street Lighting: Two Experimental Studies on Its Effects on Perceived Safety, Prospect, Concealment, and Escape," Journal of Environmental Psychology, vol. 32, no. 4, pp. 34-352, Dec. 2012.

[19] N. Davoudian and P. Raynham, "What Do Pedestrians Look At At night?" Lighting Research and Technology, vol. 44, no. 4, pp. 438-448, 2012.

[20] S. Hiranvarodom, "A Comparative Analysis of Photovoltaic Street Lighting Systems Installed in Thailand," in Proc. $3^{\text {rd }}$ World Conf. Photovoltaic Energy Conversion, Osaka, 2003, pp. 2478-2481. 